\title{
Proyección Laboral de las Estudiantes Mujeres en Carreras Mineras en la Educación Superior Chilena
}

\author{
Paulina Salinas ${ }^{(1) *}$ y Gianni Romaní(2) \\ (1) Universidad Católica del Norte, Facultad de Humanidades, Av. Angamos 0610, Antofagasta - Chile. \\ (e-mail: psalinas@ucn.cl) \\ (2) Universidad Católica del Norte, Facultad de Economía y Administración/Centro de Emprendimiento y de \\ la Pyme, Antofagasta - Chile (e-mail: gachocce@ucn.cl)
}

${ }^{*}$ Autora a quién debe dirigirse la correspondencia

Recibido Oct. 19, 2016; Aceptado Dic. 22, 2016; Versión final Ene 9, 2017, Publicado Jun. 2017

\begin{abstract}
Resumen
Este artículo analiza las percepciones de las mujeres que cursan carreras mineras en la educación superior en Chile, sobre la proyección laboral y las condiciones de empleabilidad que ofrece el sector minero. A través de un estudio descriptivo, comparativo y una metodología cualitativa, basada en 59 entrevistas a mujeres estudiantes de carreras mineras, se analizaron las motivaciones, visiones y expectativas laborales en la industria. Los resultados evidencian las tensiones que perciben las jóvenes desde el inicio hasta el término de su formación profesional, por la hegemonía masculina del rubro, la incertidumbre por compatibilizar la maternidad y el trabajo, y la inequidad que perciben entre los años que estudian y los obstáculos laborales que enfrentan. La implicancia del estudio es abordar la formación de género desde la educación superior y con ello mejorar la proyección laboral de las mujeres en mercados laborales masculinos, cuestión pendiente tanto a nivel nacional como latinoamericano.
\end{abstract}

Palabras clave: proyección laboral; género; carreras mineras; educación superior; mujeres estudiantes

\section{Employment Projection of Women Students in Mining Courses in the Chilean Higher Education System}

\begin{abstract}
:
This article analyzes the perceptions of higher education female students enrolled in mining courses in Chile about their employment expectation and employment conditions offered by the mining industry. The motivations, visions and employment expectations in the mining industry were analyzed through a descriptive, comparative and qualitative methodology based in 59 interviews with thematic focus to mining courses students. The results show the tensions that female students perceive from the beginning to the end of their courses, due to male hegemony that dominates the industry, the uncertainty to combine motherhood and work, the inequity perceived and the labor obstacles they face. The implication of the study is to address gender training from higher education and thereby improve labor projection of women in male labor markets. This aspect is a pending task at both national and Latin American level.
\end{abstract}

Keywords: employment projection; gender; mining courses; higher education; female students 


\section{INTRODUCCIÓN}

La proyección laboral de las mujeres en el mercado laboral minero y los aspectos que rodean su desarrollo es un tema central, a la luz del art. 4 de la declaración mundial sobre la Educación Superior en el siglo XXI de la UNESCO, donde se establece la relevancia de tener en cuenta el punto de vista de género en las distintas disciplinas, y consolidar la participación cualitativa de las mujeres en todos los niveles, sobre todo en las áreas en que están insuficientemente representadas (Unesco, 1998). Aunque la minería es un ejemplo de aquello, son escasos los estudios en América Latina en general y, en Chile en particular, que abordan desde la perspectiva de las estudiantes específicamente, las brechas existentes entre sus proyecciones laborales y las condiciones de empleabilidad que perciben en este rubro de la economía, puesto que por muchos años el ingreso de las mujeres a las faenas mineras estuvo prohibido, tanto por mito como por ley, siendo abolida en el año 1996 la ley que prohibía el ingreso de las mujeres a las minas subterráneas (Ayala, 2012).

Sin embargo, algunos estudios más cercanos a la temática están relacionados con las barreras de carrera percibidas por las estudiantes, estas barreras han sido definidas como eventos o condiciones, dentro de la persona o su entorno, que hacen difícil el progreso de la carrera, incluyendo una diversidad de factores internos y externos (Swanson y Woitke, 1997). En Portugal en un estudio con 457 estudiantes, identificaron el impacto de las variables socioeconómicas, raciales y étnicas en grupos minoritarios, entre ellos las mujeres, constituyéndose en obstáculos en el progreso de carrera (Cardoso y Marques, 2008). Centrándonos en la variable de género, las investigaciones son coincidentes, Bester (2011) en su estudio realizado en Sudáfrica con estudiantes universitarias, concluye que las mujeres son más proclives a recibir comentarios negativos o a experimentar conductas de discriminación sexual.

En lo específico, en Chile se desconocen las percepciones sobre la formación que reciben las mujeres en el sistema de educación superior y su futuro laboral. Hasta ahora las investigaciones no abordan los diferentes impactos que este fenómeno tiene, en los procesos de incorporación, adaptación y proyección de ellas en la industria, ni tampoco los costos económicos, sociales y familiares que provoca su menor participación en el sector. Respecto a la participación de las mujeres en la industria minera, aunque los registros son escasos, de acuerdo al reporte de la Organización Internacional del Trabajo, OIT (2014), México tiene un 13,6\% de mujeres en el rubro, Colombia, $5 \%$, Perú $2 \%$ y Chile, $7,2 \%$. Por lo tanto, se trata de una participación aún muy inicial en la región, en contraste con las cifras que reporta, esta misma fuente, para Australia y Canadá con $15 \%$ y $16 \%$ respectivamente.

Otros antecedentes necesarios de tener en cuenta en el marco de esta problemática, se relacionan con que primero, Chile es el país de la OCDE con menor participación laboral femenina; las cifras bordean el $43 \%$ contra el 54,1\% que promedian los países del bloque (Instituto Nacional de Estadística, 2013; OCDE, 2015); segundo, la minería del cobre es el sector productivo central de la economía chilena, no obstante, sólo un $7,2 \%$ de mujeres participa en el rubro; tercero, la minería es la actividad económica que menos empleo femenino otorga; por lo tanto, es un objetivo para el sector público y privado incrementar en $10 \%$, al menos, la dotación de mujeres hacia el 2023 (Área Minera, 2015). Según un informe del Consejo Minero Chileno (2014), hay diversas razones para revertir la menor presencia femenina en el rubro. Primero, es una medida para enfrentar el déficit de capital humano proyectado para el sector, que asciende a 27 mil puestos de trabajo hacia el 2023; segundo, la actividad minera posibilita que los trabajadores tengan acceso a mejores empleos con mayores beneficios y remuneraciones; tercero, mejorar la calidad de vida y la profesionalización del sector femenino, por ser una industria intensiva en capacitación y perfeccionamiento; y cuarto, el aumento de las mujeres en la minería permite romper con círculos de pobreza. En 2013, la proporción de jefas de hogar alcanza un 37,9\% en el país (Encuesta de Caracterización Socioeconómica Nacional, CASEN, 2013).

No obstante, en los últimos años, las sociedades occidentales han experimentado importantes avances respecto a la igualdad de oportunidades entre hombres y mujeres en distintos ámbitos. En algunos casos, los logros han sido notables, pero en otros el principio de igualdad de oportunidades y de no discriminación, resulta sólo un criterio legal, pero no un hecho plenamente conseguido (Morales y Cuadrado, 2007). La distribución de género en la Educación Superior en el país es un ejemplo en este sentido, ya que las mujeres participan en un 51,8\%; aunque mayoritariamente en carreras típicamente femeninas, en cambio en las típicamente masculinas y orientadas al rubro minero, (Ingeniería Civil Industria, Civil Metalurgia, Civil en Minas, Geología, Civil en Construcción e Ingeniería en Prevención de Riesgo) la presencia alcanza sólo un $23 \%$, acentuándose esta asimetría en las carreras técnicas (Electricidad, Electrónica, Automatización y Control Industrial, Técnico en Minería y Geominería) donde participa sólo un 4,3\% (Servicio de Información de Educación Superior, SIES, 2014). 
Una distribución levemente mejor, muestra México, con el $71 \%$ para los hombres y el $29 \%$ mujeres en carreras del área minera y extracción como: Ingeniería petrolera y de gas, Ingeniería geológica, Ingeniería de minas, Tecnología de minerales y Extracción de materias primas (Mi Futuro, 2014). En cambio, para los hombres en Chile, las carreras técnicas y profesionales del área de la minería lideran el ranking de empleabilidad 2014. Así lo indican las estadísticas del portal Mi Futuro, del Mineduc, las que posicionan a Ingeniería Civil en Minas e Ingeniería en Minas y Metalurgia como las dos carreras universitarias con mejor proyección laboral (Mineduc, 2016). En el caso de las mujeres, la menor presencia de ellas en estas carreras redunda en una inferior ocupación posteriormente en el mercado laboral de la industria (Consejo Minero Chileno, 2012).

Bajo este escenario, se advierte que el sistema de educación superior requiere transformaciones para fortalecer el acceso y proyección femenina en las carreras para la minería, así como para la posterior incorporación a mercados laborales de predominio masculino. A partir de estos antecedentes, el objetivo que se propone en este trabajo es analizar las percepciones de las mujeres, que cursan carreras mineras en la educación superior, sobre la proyección laboral y las condiciones de empleabilidad que ofrece el rubro. Para ello se buscó responder interrogantes como ¿cuáles son las motivaciones, visiones y expectativas laborales de las estudiantes de carreras mineras de nivel universitario y técnico sobre su futuro laboral en la industria? ¿Qué diferencias y/o semejanzas se hallan en sus discursos? ¿Cuáles son las tensiones que enfrentan para proyectar su futuro laboral en la minería? ¿Pueden proyectar su futuro laboral en la industria? La hipótesis teórica se relaciona con que existen diferencias entre las proyecciones laborales de las estudiantes mujeres que cursan carreras en el área minera y las condiciones de empleabilidad que prospectan en este sector. Por lo tanto, la implicancia del estudio es aportar evidencia para mejorar la proyección laboral de las mujeres en mercados laborales masculinos, cuestión pendiente tanto a nivel nacional como latinoamericano. En este trabajo teorizamos en primer lugar sobre la elección de carrera y la proyección laboral desde el género, las barreras de carrera y sobre género y mercado labora

\section{ELECCIÓN DE CARRERA Y PROYECCIÓN LABORAL DESDE LOS CONSTRUCTOS DE GÉNERO}

La literatura a nivel internacional ha sido extensa y coincidente respecto a que la elección de una carrera típicamente femenina o masculina se relaciona con múltiples factores relacionados con la socialización de mujeres y hombres en el sistema familiar y escolar; ambos son los mecanismos de mayor influencia sobre el comportamiento personal y social del individuo, en el caso de España lo confirma el estudio de Sáinz, López-Sáez y Lisbona (2004), y en un estudio realizado en Colombia por Espejo (2015). También esta elección se relaciona con las competencias personales como: la cualificación, es decir, la pericia y conocimiento sobre lo que la persona sabe; el talento, que está asociado con aquellas habilidades, destrezas, capacidades de índole general o específico; y finalmente, con el talante, esto es la voluntad, motivación, actitudes y valores (Levy-Leboyer, 1997; Moriano, Palaci y Morales, 2006).

López-Sáez $(1995,2004)$ plantea que las diferencias entre hombres y mujeres en la elección de carreras, está determinada por los patrones culturales de género, incorporando en su modelo variables psicosociales como el Auto-concepto de Género y las Normas Culturales. Los estereotipos que existen sobre ambos sexos afectan al esquema del yo, en los valores personales y en las características estereotipadas asociadas a cada carrera. Un supuesto compartido por las teorías y modelos que describen la conducta de las personas frente a la elección de carrera, es la elaboración de una serie de creencias o expectativas de resultado asociado a cada alternativa (proyección laboral), por quien hace la elección, donde el logro esperado tiene una importante valoración (López-Sáez, 1995). Por tanto, se escogen aquellas alternativas en las que el sentimiento de competencia resulta satisfactorio para el postulante (Lisbona y Topa, 2003).

Algunas de las teorías desarrolladas respecto a la elección de carrera se vinculan con aquellas basadas en el Autoconcepto o Autoconfianza; con la Atribución; la Orientación a la Superación o la Indefensión Aprendida y la Expectativa-Valor (López-Sáez, 1995). La primera, Autoconcepto, se refiere a la evaluación de la propia capacidad del sujeto para realizar tareas específicas o para llevar a cabo las conductas apropiadas para cada función (Eccles, Adler, Futterman, Goff, Kaczala, Meece y Midgley, 1983), siendo uno de los factores que influye en la elección de carreras. Por tanto, en las decisiones académica-profesional de mujeres y hombres existen diferencia que son asociadas al género (Mosterio, 1997).

En estrecha relación con esta teoría, se encuentra el concepto de Autoeficiencia, introducido por Bandura (1977, citado por Mosterio, 1997) donde se explica la valoración que realiza una persona de su capacidad de rendimiento. Hombres y mujeres tienen una correspondiente autoeficiencia respecto a las ocupaciones tradicionales según su sexo. Ellas se sienten más capaces en actividades asociadas a su rol tradicional y tienen más temor de fallar en las labores no tradicionales. 
La segunda teoría, de la Atribución, explica la diferencia en los estilos atributivos de hombres y mujeres. En relación a los fracasos, ellos los atribuyen a factores externos e inestables, mientras que las mujeres a circunstancias internas y estables. En cambio, la atribución a los éxitos por parte de los varones se vinculan a factores internos y estables; por el contrario, las mujeres lo asocian a causas externas e inestables (López-Sáez, 1995).

La tercera, la teoría de la Orientación a la Superación o a la Indefensión Aprendida, se relaciona con los estilos atributivos antes mencionados. De este modo, la diferencia en la elección de carrera está basada en la debilidad frente a los fracasos que anticipan las mujeres, y la tendencia a la superación que prima en el caso de los hombres. Por último, el Modelo de Expectativa-Valor, explica la elección de tareas y de una ocupación, respectivamente. En este sentido, hay diferencias entre hombres y mujeres en la forma de valorar los beneficios que se asocian al cumplimiento de un determinado rol (Lisbona y Topa, 2003). Las expectativas de éxito y la valoración de las tareas explican los motivos por los cuales las personas llevan a cabo una determinada elección. No obstante, las expectativas de éxito como la valoración, no están libres de los roles de género ni de las presiones sociales y culturales prevalecientes (Sáinz, et. al., 2004).

Otro modelo relacionado con la elección de carrera es el de Elección de Logro. Este patrón propone que "tanto los acontecimientos pasados vividos, como las experiencias previas de éxito o fracaso, y otros factores culturales ejercen un efecto indirecto sobre las elecciones que los sujetos realizan. Este proceso está mediado por las interpretaciones personales que los individuos hacen de las experiencias vividas, por la percepción de las expectativas de otros, y por el proceso de identificación con las metas y valores en torno a los roles prevalecientes en el contexto sociocultural” (Eccles, et. al., 1983: 82).

\section{Barreras de carrera}

Las barreras de carrera fueron definidas por Swanson y Woitke (1997:446), como "eventos o condiciones, ya sea dentro de la persona o en su entorno que hacen difícil el progreso de la carrera". Se trata de una amplia gama de obstáculos que pueden interferir en el desarrollo de la carrera profesional de una persona.

Diversos son los estudios que han abordado las barreras de carrera, que frenan la incorporación al mercado laboral o impiden el desarrollo profesional de las mujeres. Swanson, Daniels y Tokar (1996), identificaron barreras como: discriminación sexual, falta de confianza, conflicto de rol, conflicto entre los hijos y las exigencia profesionales, discriminación racial, dificultades en la toma de decisiones, falta de modelos o mentores, entre otras. En esta línea Cardoso y Marques (2008) coinciden en la existencia de barreras socioeconómicas, étnicas o raciales y de género.

La variable de género es una importante moderadora en el desarrollo profesional de las personas, por lo mismo la discriminación sexual ha sido una constante en diferentes contextos laborales. Donoso, Figuera y Rodríguez Moreno (2011) en una investigación con estudiantes universitarios españoles, concluyen que las universitarias presentan elevadas metas profesionales, pero buscan compatibilizar el trabajo con otros roles vitales. En relación a las aspiraciones de futuro, se posicionan a favor de un liderazgo de tipo experto frente a un liderazgo empresarial; quizás, por ello no están inclinadas a crear redes sociales con fines exclusivamente profesionales y abogan por redes más cooperativas. También, la evaluación negativa se identifica como la barrera que más condicionará el progreso profesional de la mujer universitaria. Respecto a este punto, explican que las mujeres manifiestan mayor miedo hacia esta evaluación, hecho estrechamente relacionado con la preocupación por las críticas de los otros y la percepción de que a ellas se les perdonan menos los errores, especialmente si ocupan un puesto de poder.

Navarro, Román e Infante (2016) hicieron una revisión exhaustiva a nivel internacional identificando la diversidad de estudios que han abordado la temática sobre las barreras de carrera reales, refiriéndose al área de la construcción. Al respecto destacan las siguientes: la maternidad, la conciliación y el rol múltiple, los estereotipos de género, la promoción, las condiciones de trabajo, la cultura machista, la asignación de puestos, el acoso y la falta de respeto, el reclutamiento y la selección, la retribución, las redes sociales, el desempeño y las propias mujeres. Sin embargo, de acuerdo a lo planteado por estas autoras estas barreras coinciden con las barreras percibidas, que son aquellas reconocidas por las estudiantes que aunque no tienen experiencia en el sector, anticipan las dificultades que pueden encontrar en el desarrollo de la profesión. Se reiteran la maternidad y multiplicidad de roles, los estereotipos de género, el acoso y falta de respeto, entre otras. En palabras de Olavarría y Céspedes, (2002) las mujeres enfrentan la necesidad de construir la vida personal a través de representaciones colectivas contradictorias, esto es, entre la madre full time y la maternidad part-time. Entre ambas, los devaneos son constantes y la tensión interiorizada patente. 
En la mayoría de las investigaciones, las estudiantes tienen conciencia de los obstáculos que les esperan en el mercado laboral, sobre todo en aquellos de predominio masculino. Por lo tanto, estos aspectos son esperables en la industria minera, por la fuerza de la hegemonía masculina del rubro que condiciona la cultura organizacional y la menor presencia de las mujeres en el sector (Salinas y Romaní, 2016; Ayala, 2012).

\section{Género y mercado laboral minero}

El género se asocia con un sistema de poder que circula en términos universales y que está presente bajo la misma lógica en todos los ámbitos de la vida de las mujeres, estableciendo jerarquías y asimetrías entre los sujetos (Ramos, Sarrio, Barberá y Candela, 2002; Benitez, 2012; Painter-Morland, 2012). Scott (1986) señala que el género es un elemento constitutivo de las relaciones sociales, basado en las diferencias percibidas entre ambos sexos. Según esta autora, el género comprende cuatro elementos interrelacionados: primero, los símbolos culturales, que son representaciones múltiples y a menudo contradictorias; segundo, los conceptos normativos que expresan interpretaciones del significado de estos símbolos, frecuentemente como oposiciones binarias (fuertes/débiles, activos/pasivas); tercero, los sistemas de parentesco, mercado laborales, sistemas educativos y la organización política; y cuarto, las identidades subjetivas. Harding (1986) agrega el carácter multifacético y desconstructivista del género, identificando tres niveles importantes: el simbolismo de género, la división del trabajo y las identidades y deseos marcados por esta variable. En consecuencia, el constructo género es individual, estructural, simbólico y asimétrico.

Desde las teorías del mercado laboral, se ha estudiado la segregación de género en aquellos espacios de la estructura ocupacional donde el empleo se considera conforme a la "naturaleza femenina", como una extensión de sus responsabilidades domésticas, en trabajos con escasa estabilidad, compatibles con el cuidado de los hijos, baja productividad y reducidos salarios. Es una fuerza de trabajo secundaria, cuyo salario es un suplemento del ingreso del hombre (Espino, 2012).

El trabajo remunerado femenino se pondera en un rango inferior al masculino, porque la conducta profesional de ellas se distingue debido al proceso de socialización y, por la propia experiencia, en el trabajo doméstico, de manera que las mujeres arrastran su inferioridad de estatus al puesto de trabajo (UribeEcheverría, 2008). Así, la segregación ocupacional por sexo constituye un aspecto estructurante, ya que no responde a la ineficiente asignación de recursos laborales a ciertas áreas, sino que gran parte de las diferencias salariales se deben a que el sector femenino se concentra en ocupaciones con menor remuneración (Ibañez, 2010). En particular en la minería, existen barreras que dificultan el ingreso de las mujeres al mercado laboral y que pueden, inclusive, ser un impedimento para el desarrollo del sector privado y el crecimiento económico de un país (International Women and Mining Network (IWIM), 2014).

Estas barreras se relacionan con la distribución histórica por género del trabajo y la elaboración de estereotipos, específicamente la auto discriminación de las mujeres, las responsabilidades familiares y la hegemonía masculina. La primera, se percibe de distinta forma entre hombres y mujeres. Ellos la explican como una falta de confianza de las mujeres respecto a la creencia de ser capaces de trabajar en minería; en cambio ellas, plantean que la auto discriminación es provocada por las diferencias de género que coexisten en el rubro (Salinas y Romaní, 2014). Respecto a la segunda y tercera barrera, hay percepciones similares entre hombres y mujeres sobre las responsabilidades familiares y la hegemonía del varón dentro de la industria, pues se asocia a la mujer el rol central de madre y se le asigna el peso de la familia. A la vez, la hegemonía masculina en el sector favorece las prácticas discriminatorias (Salinas, 2013).

Pese a estos antecedentes el IWIM (2014), afirma que contratar personal femenino trae múltiples beneficios a las comunidades, ya que cuando las mujeres tienen acceso al trabajo y, por tanto al poder sobre las finanzas del hogar, son más propensas a invertir en educación y salud. Además se revierte la dependencia económica que limita la influencia de ellas en las decisiones del grupo familiar (Arraigada, 2014).

\section{METODOLOGÍA}

De acuerdo a los objetivos del presente estudio se utilizó un enfoque cualitativo, adscribimos a una concepción investigativa que asume de que la realidad social es subjetiva, múltiple y cambiante, es el resultado de una construcción de los sujetos participantes que se encuentran en interacción con otros miembros de la sociedad, de este modo el interés se centra en comprender e interpretar las percepciones de las estudiantes que cursan carreras mineras en la educación superior, sobre la proyección laboral y las condiciones de empleabilidad que ofrece el rubro, según como es entendida por sus protagonistas (Salinas y Cárdenas, 2009; Denzin y Lincoln, 2012). Por lo tanto, el diseño de investigación se enmarca en un estudio descriptivo con fines comparativos. 


\section{Instrumento de recogida de información y procedimiento}

Se aplicaron entrevistas en profundidad de carácter individual, ya que las características dialógicas de esta técnica permitió acceder a las percepciones de las estudiantes, y el carácter individual fue el más apropiado para acceder a la unidad de análisis. Se trata de jóvenes que cursan carreras técnicas y/o universitarias, en los distintos establecimientos de educación superior ubicados en Antofagasta, epicentro de la minería del cobre a nivel mundial. Las dificultades de acceso a la muestra, debido a la carga curricular de las participantes, determinaron que esta técnica fuera la más apropiada, para el logro de los objetivos del estudio. Las entrevistas se desarrollaron en forma secuencial, de tal manera que una vez que la primera fue realizada se procedió a su trascripción y análisis preliminar, de este modo la segunda consideró las observaciones o conclusiones previas, y que fueron importantes de incorporar en la segunda entrevista, y así sucesivamente se desarrolló la fase de recopilación de la información.

Las entrevistas abordaron tres ejes temáticos centrales 1) percepción de carrera, 2) incorporación de la mujer en carreras masculinas y 3) expectativa frente a su futura inserción laboral. Para cada entrevista se solicitó la autorización de la participante, a través de un consentimiento informado, asegurando la aceptación y confidencialidad de su identidad (criterio de validación). Las entrevistas fueron realizadas entre mayo y agosto 2014. Fueron grabadas previo consentimiento informado, luego transcritas en forma textual y tuvieron una duración entre 60 y 90 minutos.

Se entrevistaron estudiantes hasta saturar (criterio de validación) la información, es decir la reiteración de los discursos, punto que fue alcanzado con un total de 59 jóvenes, que cursan carreras de nivel técnico y universitario, ambas modalidades son las que tiene el sistema de educación chileno para la formación profesional. Las entrevistadas fueron contactadas directamente en sus universidades o Centros de Formación Técnica, y el dato de una permitió acceder a la siguiente y así sucesivamente (bola de nieve). Además se triangularon (criterio de validación) los discursos de las estudiantes de nivel técnico con las universitarias.

\section{Descripción de la muestra}

De acuerdo al enfoque del estudio se definió un muestreo intencional (Ruiz, 1996), seleccionando a estudiantes de carreras vinculadas a la industria minera que cursan el primer año y de este modo conocer las motivaciones que las llevaron a optar por esta área, y a la vez a estudiantes que cursaban los últimos dos años de sus carreras, para identificar las expectativas laborales que tenían, dada su cercanía con el mercado laboral. Las carreras universitarias tienen en promedio entre 4 y 6 años de duración, mientras que las carreras técnicas (Centro de Formación Técnica, CFT) tienen en promedio dos años.

Se entrevistaron 59 estudiantes, 19 cursan el primer año de una carrera universitaria y 14 de carácter técnica. En el último año de estudio, se entrevistaron 16 universitarias y 10 técnicas. 19 años es la edad promedio de las alumnas de primer año universitario y 24 años en las que cursan el nivel técnico. 24 años en el caso de las universitarias que cursan el último año de la carrera, y 26 años la edad promedio de las técnicas (ver tabla 1).

Tabla 1: Características de la Muestra

\begin{tabular}{|l|c|c|}
\hline & Universitarias & \multicolumn{1}{|c|}{ Técnicas } \\
\hline Primer año (n) & 19 & 24 \\
\hline Edad Promedio (Primer año) & 19 & 10 \\
\hline Último año (n) & 16 & 26 \\
\hline Edad Promedio (último año) & 24 & \multicolumn{1}{|c|}{} \\
\hline Carreras & $\begin{array}{l}\text { Geología, ingeniería civil; ambiental, } \\
\text { metalurgia y Minas, Química y } \\
\text { Geomática, Civil Industrial, Ingeniería } \\
\text { de Ejecución Metalúrgica y mecánica. }\end{array}$ & $\begin{array}{l}\text { Técnico en Minería, Explotación minera, } \\
\text { Maquinaria Pesada, Automatización y } \\
\text { control Industrial, Mecánica Automotriz } \\
\text { en Sist. Elect. Y Mantenimiento Industrial }\end{array}$ \\
\hline Instituciones & $\begin{array}{l}\text { Universidad Católica del Norte(UCN), } \\
\text { Universidad de Antofagasta (UA) }\end{array}$ & $\begin{array}{l}\text { Centro de Educación y Capacitación de } \\
\text { la Universidad Católica del Norte, } \\
\text { (CEDUC), AlEP de la Universidad } \\
\text { Andrés Bello, INACAP (Instituto } \\
\text { Educación Superior Técnica). }\end{array}$ \\
\hline
\end{tabular}




\section{Análisis de la información}

El análisis de la información se realizó desde la perspectiva social/hermenéutica, cuyo énfasis está en las construcciones simbólicas del discurso que debe sus propiedades más específicas a las condiciones sociales de producción (Alonso, 2003). Para ello, se realizó una lectura detallada línea a línea de cada una de las entrevistas, buscando reducir el extenso material que se recopiló, en base a una versión simplificada de la propuesta de codificación de la Teoría Fundamentada (Strauss y Corbin 2002; Trinidad et al. 2006). Se identificaron las categorías abiertas, esto implicó desarrollar un procedimiento analítico por medio del cual se reconocen los conceptos y se descubren en los datos, sus propiedades y dimensiones. A partir de ello, se distinguieron las sub categorías. Una vez registrado estos dos niveles de codificación, se establecieron las categorías centrales que equivalen a un mayor grado de análisis. Este procedimiento fue adecuado para la presente investigación, ya que generó resultados que describen el fenómeno en estudio y permiten establecer relaciones e identificar los aspectos del proceso.

\section{RESULTADOS}

De acuerdo al objetivo y análisis de las entrevistas realizadas, los resultados se agrupan en tres categorías centrales:1) Motivaciones para ingresar a carreras del área minera, 2) Visión sobre la carrera y 3) Expectativas sobre el futuro laboral

\section{Motivaciones para ingresar a carreras del área minera}

En esta primera categoría central sobre las motivaciones, los discursos de las estudiantes muestran similitudes entre aquellas que cursan primer año de nivel técnico y/o universitario, pero también algunas diferencias. En lo específico, la Familia es la primera motivación asociada al área minera, pues ser hijas de padre minero o hermana, produce un estrecho vínculo con el rubro y su cultura. Es decir, la socialización del sistema familiar vinculada al sector es un eje central en la proyección laboral de las estudiantes que eligen carreras en el área. Pero también, están los casos inversos donde la familia no apoya el interés temprano de la hija en la minería, por el hecho de ser mujer e iniciar una carrera en este rubro, provocando el efecto inverso en las estudiantes, a pesar de la desaprobación familiar.

"Tengo un hermano por parte de mamá, él es ingeniero en maquinaria pesada y salió de aquí, de INACAP... me tiene fe... entonces me motivó y fue como un ejemplo a seguir" (Tecnología en Minería, 19 años).

"El hecho de que (familia) no hayan querido que yo estudiara eso, me ayudó a que dijera: No, es lo que yo quiero, tengo que hacer lo que a mí me gusta y no lo que mi papá y mis abuelos digan..." (Geología, 18 años).

"Cuando yo recién empecé a estudiar mis abuelitas decían "pero cómo si hay puros hombres", "si es muy difícil" (Geología, 19 años).

La segunda motivación identificada en las entrevistadas corresponde a las Competencias Personales, es decir, la pericia y el conocimiento, lo que las jóvenes saben de sí mismas, donde ellas evalúan sus propias capacidades, que en el caso de las carreras mineras, están orientadas a las matemáticas, a la ciencia y a los procesos mineros. Los discursos a continuación lo expresan:

"Siempre he sido buena para las matemáticas... y eso es un beneficio, aparte la geología es súper matemática y científica a la vez entonces esa es una ventaja” (Geología, 18 años).

"La carrera de nosotros tiene todo lo que es matemáticas, física, entonces como que yo encuentro que mi fortaleza es esa, porque tengo los conocimientos aún ahí intactos, fresquitos" (Operación de Plantas Mineras, 19 años).

"Lo primero que me motivó a seguir el curso fue el tema de los procesos mineros, me metí mucho en el tema, me gustó." (Operación de Plantas Mineras, 19 años)

Una tercera motivación, relacionada con la anterior, está vinculada con el temprano interés que identifican las estudiantes de primer año por el Área Minera. No obstante, las universitarias lo expresan de manera más general y abstracta: "gusto por el área industrial", "interés por innovar y aprender nuevas tecnologías" y "gusto por trabajo al aire libre". En cambio las técnicas expresan motivaciones más concretas: "tomar muestras", "salir a terreno", "interés por análisis metalúrgico", "interés por proceso mineros". Estas distinciones se asocian con las diferencias socioeconómicas y culturales que existen entre ambos grupos, pero a la vez esbozan las transformaciones de género, que han ocurrido en los últimos años en algunos sectores de la sociedad chilena, ya que estas jóvenes amplían sus áreas de interés a sectores no tradicionales y están dispuestas a enfrentar los estereotipos de género que priman en la elección de carrera, revirtiendo los modelos tradicionales de socialización familiar. 
"Mi papá siempre tuvo auto, entonces él lo arreglaba en la casa y me acuerdo que se metía abajo del auto y yo me metía con él y preguntaba papá qué es esto y cómo funciona y ahí me empezó a gustar, pero fue hasta como los 12 años (...) Yo misma me preguntaba de donde saqué mecánica y después pensando, claro eso influyó, esos 5 años en que yo pasaba debajo del auto" (Mecánica Automotriz y Sistemas Eléctricos, 19 años).

La cuarta motivación identificada en los discursos de las estudiantes se refiere al aspecto Económico. La alta rentabilidad que ofrece el rubro es un incentivo importante para elegir una carrera del sector minero; al respecto, existe coincidencia en ambos grupos analizados: "independencia económica", "interés por las remuneraciones del sector", "sustentabilidad económica de la carrera", "interés por carrera rentable" y "bienestar económico". Las estudiantes técnicas agregan en este ámbito el aporte que conlleva el beneficio económico para los hijos: "satisfacer las necesidades de la familia".

"Yo creo que todos los que estamos estudiando esta carrera nos lleva un poco el gusto por lo que supuestamente se va a pagar" (Ingeniería Civil de Minas, 22 años).

"El sueldo, como todos saben tiene mucha rentabilidad, una carrera en Antofagasta donde puedes surgir, donde hay trabajo, nunca va a estar limitado el trabajo en ese sentido” (Ing. Ejecución Metalurgia, 19 años). "[...] la vi por las lucas (dinero), hay harto campo acá en la segunda región. Yo sola con mi hijo y tener mis cosas... que a mi hijo no le falte nada, eso es como lo primordial" (Tecnología en Minería, 19 años).

Por último, la Duración de la Carrera es otra de las motivaciones mencionadas. Sin embargo, en este aspecto las estudiantes de CFT son más precisas en señalar las ventajas de optar a carreras de nivel técnico: "son carrera más corta de nivel superior", "es un escalafón para alcanzar una ingeniería" y "la práctica para obtener experiencia y conocimiento".

"Al principio dije no, no puedo con esto o sea yo me matriculé enfocada en sacar la carrera en los dos años o sea ni echarme los ramos, ni dar exámenes ni nada, bueno gracias a Dios lo logré, pero si me costó mucho, porque no sabía nada de mecánica o sea no tenía ni una base de nada con suerte sabía cómo funcionaba un auto así te juro, fue como frustrante al principio" (Maquinaria Pesada, 22 años).

Destacan la flexibilidad que ofrece este tipo de formación: a) las salidas intermedias (primero un grado técnico a los dos años y luego, opcional, dos años más para obtener el grado de ingenieras); b) las horas de práctica como vínculo directo con la empresa, y c) el estatus de formación superior. En cambio las estudiantes universitarias resaltan que al menos existe la posibilidad que en cuatro años obtengan el título de ingeniera en ejecución. Esta diferencia muestra las brechas que hay entre uno y otro sistema de formación profesional y las escasas modificaciones que ha tenido, en este sentido, el sector universitario en el país, predominando el currículum entre de 10 y 12 semestres inclusive. A continuación la tabla 2 sintetiza los resultados obtenidos.

Tabla 2: Estudiantes universitarias y técnicas de I año en carreras mineras

\begin{tabular}{|l|l|l|}
\hline \multirow{2}{*}{\begin{tabular}{l} 
Categoría abierta- $\begin{array}{l}\text { Categoría central: motivaciones para cursar carreras del área minera } \\
\text { conceptual }\end{array}$ \\
\cline { 2 - 3 }
\end{tabular}} & $\begin{array}{l}\text { Universitarias } \\
\text { primer año } \\
\text { subcategoría }\end{array}$ & $\begin{array}{l}\text { Técnicas } \\
\text { primer año } \\
\text { subcategoría }\end{array}$ \\
\hline Familia & $\begin{array}{l}\text { Provengo de una familia minera } \\
\text { Vengo de un entorno minero }\end{array}$ & $\begin{array}{l}\text { El ejemplo de mi hermano ingeniero } \\
\text { El ejemplo de mi padre ingeniero en minas }\end{array}$ \\
\hline $\begin{array}{l}\text { Habilidades } \\
\text { científicas }\end{array}$ & $\begin{array}{l}\text { Tengo habilidades para las ciencias } \\
\text { Me gustan las matemáticas }\end{array}$ & Habilidades teóricas, física, matemática. \\
\hline $\begin{array}{l}\text { Interés por área } \\
\text { minera }\end{array}$ & $\begin{array}{l}\text { Gusto por el área industrial } \\
\text { Interés por innovar y aprender nuevas } \\
\text { tecnologías } \\
\text { Gusto por trabajo al aire libre }\end{array}$ & $\begin{array}{l}\text { Tomar muestras } \\
\text { Salir a terreno } \\
\text { Interés por análisis metalúrgico } \\
\text { Interés por proceso mineros }\end{array}$ \\
\hline Económica & $\begin{array}{l}\text { Independencia económica } \\
\text { Las remuneraciones del sector } \\
\text { Sustentabilidad económica de la carrera }\end{array}$ & $\begin{array}{l}\text { Interés por carrera rentable } \\
\text { Bienestar económico } \\
\text { Satisfacer las necesidades de la familia }\end{array}$ \\
\hline $\begin{array}{l}\text { Duración de la } \\
\text { carrera }\end{array}$ & $\begin{array}{l}\text { Alcanzar en cuatro años el título de } \\
\text { ingeniera en ejecución }\end{array}$ & $\begin{array}{l}\text { Carrera más corta de nivel superior } \\
\text { Escalafón para alcanzar una ingeniería } \\
\text { Práctica para obtener experiencia y } \\
\text { conocimiento }\end{array}$ \\
\hline
\end{tabular}




\section{Visión sobre la carrera}

La segunda categoría central corresponde a Visión sobre la Carrera, o sea, la proyección laboral que tienen sobre ella. En ambos grupos (universitarias y técnicas) hubo coincidencia en valorar positivamente la Alta Empleabilidad de la Minería. Esta apreciación se observa desde el inicio al término de la carrera, destacando la rentabilidad, las proyecciones y diversidad de opciones laborales del sector, así como los altos ingresos. Además, las estudiantes técnicas aspiran a tener un futuro mejor.

Aunque el estudio no consideró, en esta etapa, la valoración de estudiantes varones, es interesante constatar que las mujeres con esta valoración positiva, estarían contrarrestando la teoría basada en la Expectativa -Valor, donde se plantea que hay diferencias en la valoración que hacen hombres y mujeres de acuerdo al rol que desempeñan (Lisboa y Topa, 2003), cuestión que al menos en esta categoría no se estaría cumpliendo, pues para las estudiantes el aspecto económico es, en la actualidad, un factor relevante; más aún con el aumento de la jefatura femenina en los hogares.

"El sueldo, como todos saben, tiene (en la minería) mucha rentabilidad, una carrera en Antofagasta donde puedes surgir, donde hay trabajo, nunca va a estar limitado el trabajo en ese sentido" (Ing. Ejecución Metalurgia, 21 años).

"Siempre me veo independiente, de querer tener yo mi trabajo, mis cosas y no estar, dependiendo de alguien, de una pareja..." (Ingeniería Civil Ambiental, 18 años)

"Yo creo que todos los que estamos estudiando esta carrera nos lleva un poco el gusto por lo que supuestamente se va a pagar" (Ingeniería Civil de Minas, 22 años).

"Lo que te llama es la plata, que en la minería es muy bueno, es muy rentable [...], te da una perspectiva alta para lo que tú quieres lograr, a menos en mí caso, por ejemplo yo como madre soltera es como aspirar a más, a tener un mejor futuro para mi hijo, querer lo mejor" (Maquinaria Pesada, 22 años).

La segunda categoría que emerge de los discursos se refiere a las Distinciones de Género. Al respecto se observan similitudes entre las estudiantes de primer año; técnicas y universitarias, coinciden en que cuando ingresan a la carrera: "Ios hombres tienen más capacidad para las matemáticas", "más experiencias", "más conocimiento", "se manejan mejor"; además "tienen ventaja aquellos estudiantes que provienen de un liceo técnico". Sus discursos giran en torno a los atributos que ven en los hombres respecto a ellas mismas.

En cambio, en los discursos de las estudiantes técnicas o universitarias que cursan los últimos años de la carrera, se evidencia una transformación significativa en el Autoconcepto. Manifiestan que no hay diferencias entre hombres y mujeres para desempeñarse en la minería, ya que tienen igualdad de capacidades: "son ordenadas", "responsables", e incluso aprecian "el incremento en el número de mujeres en sus carreras". No obstante, el grupo de universitarias destaca que no hay incentivos suficientes para la incorporación de mujeres en carreras mineras. Estas diferencias en los resultados permiten visualizar las transformaciones que se producen durante el proceso de formación de las estudiantes, donde se logra reforzar la autoconfianza y el desarrollo de sus capacidades. Algunos discursos que ejemplifican lo expuesto.

"Las mujeres somos más ordenadas y vamos a saber cómo hacer las cosas. Los hombres son como arrebatados, machos y por ser apurones y arrebatados a veces le salen las cosas mal" (Tecnología en Minería, 19 años)

"Como mujeres tampoco tenemos que pararnos en menos al nivel del hombre, o sea tenemos que ir al mismo nivel y que no se note la diferencia entre un hombre y una mujer, pararnos como de igual a igual" (Ing. Civil Industrial Química, 22 años).

"Se demostró, se sigue demostrando que la mujer tiene las mismas capacidades que los hombres y que pueden hacer las mismas cosas, no por ser mujer tienes que ver de como el sexo débil de que no puede ser, o que no se puede ensuciar las manos, que no puede hacer trabajos pesados no" (Maquinaria Pesada, 20 años).

"Yo creo que la mujer igual podría realizar todo lo que el hombre hace en la mina. Creo que tenemos las mismas capacidades. Quizás no se le ha dado la misma oportunidad a la mujer para que la demuestre" (Operación de Plantas Mineras, 19 años).

La tercera categoría, Hegemonía Masculina, se muestra en un sentido inverso respecto a la anterior, pues aquí hay coincidencias categóricas entre ambos grupos analizados, universitarias y técnicas de primero o 
último año. Comparten la visión sobre las carreras del área minera y el rubro en general. Al respecto, mencionan la vigencia de la cultura "machista" en dos dimensiones: la primera asociada a las características masculinas del trabajo en la minería, reconocen que se trata de un rubro donde predominan los hábitos masculinos como la fuerza física, las groserías en el vocabulario, la mayor disponibilidad para desempeñarse en el rubro, el alto riesgo del sector, entre otros.

La segunda dimensión está referida a las discriminaciones percibidas hacia ellas en tanto mujeres: "la mujer es un tabú aún en la industria", "tienen menos disponibilidad por embarazo", "se ahorran costos no contratando mujeres", "enfrentan el asedio masculino", "reciben menos remuneraciones", "prejuicios por cambios hormonales", "menstruación", "se desconfía de las intenciones de las mujeres", "se sorprenden porque estudian carreras mineras" o "porque están en carreras de hombres", entre otras. Algunos discursos que ejemplifican lo expuesto.

"No me agrada que a veces son demasiado ordinarios, tiran mucha talla doble sentido, groserías... hay que adaptarse no más" (Automatización y Control de Gestión, 21 años).

"Siempre me preguntaban y decían tú qué estudias, ingeniería electrónica y me decían: "uh electrónica pero hay muy pocas mujeres que se meten a esas carrera" y siempre me decían pero por qué" (Ingeniería Civil en Electrónica, 27 años).

La fuerza y transversalidad de estos discursos en las entrevistadas, puede ser comprendida desde la perspectiva analítica de género, que planteamos al inicio, siendo una de las barreras de carrera que se reitera a lo largo del estudio. El género se trata de un elemento constitutivo de las relaciones sociales, basado en las diferencias percibidas entre ambos sexos, como una forma primaria de significación de las relaciones de poder, expresado en símbolos culturales, representaciones múltiples y a menudo contradictorias (Scott, 1996). Por lo tanto, pese a lo determinante que son las visiones de las estudiantes sobre las carreras minera, ellas enfrentan estos constructos, que son tradicionalmente naturalizados y por ello se vuelven imperceptibles en los discursos que circulan en el rubro, sin embargo son barreras reales y aún presentes en el sector.

La cuarta categoría, Compatibilizar el Trabajo y la Familia, expresa la temprana, histórica y aún vigente tensión que enfrentan las mujeres al momento proyectar su futuro laboral, más aún en un rubro como la minería, donde el sistema de turno y la lejanía de las faenas mineras, es una conditio sine qua non de los yacimientos de cobre, en el caso de Chile. Los discursos analizados son similares en ambos grupos. No hay diferencias entre universitarias y técnicas, ni tampoco hay modificaciones entre las que inician o terminan sus carreras, lo que agudiza esta visión.

Las estudiantes manifiestan que no resulta fácil compatibilizar las demandas de la familia con el trabajo. La maternidad es lo más difícil, es una limitante armonizar ambos roles e, incluso, en forma radical las estudiantes técnicas expresan que "la maternidad es un problema, es mejor no tener hijos para mantener la independencia". Esta afirmación corrobora, que lejos de modificarse sigue "presente en la sociedad chilena la figura ideal de madre omnipresente extremadamente exigente. Convirtiéndose en una pesada sombra que debe conjugarse con condiciones estructurales que impiden la concreción de tal afán" (Araujo y Martuccelli, 2012:152). Imaginario que las estudiantes perciben como una tensión insoslayable, ya sea porque se proyectan con hijos, o porque no, en ambos casos deben enfrentar el peso de tal decisión o la contradicción que experimentan frente a la disyuntiva entre familia y trabajo. Algunos discursos que ejemplifican lo expuesto.

"Mis abuelos que son muy influyentes en mi familia (los papás de mi papá) me dijeron: 'No, que eso era una cuestión para hombres', aparte son a la antigua, súper machistas, dijeron: 'NO!, eso es para hombres, tú eres una niñita, tú tienes que estar en la casa con los niños'..." (Geología, 18 años).

"Igual quiero tener una familia y quiero tener hijos, el problemas es que es muy injusto, porque uno estudia prácticamente 10 años en la universidad para poder desarrollarse, para poder...porque tú después cuando empiezas a trabajar, empiezas a ver el mundo y quieres conocerlo más pero después tú dices y uno también está viendo su reloj biológico, entonces uno pucha ya dice que no se te vaya a pasar [...], cómo lo voy a poder compatibilizar, porque yo también quiero trabajar, por algo estudié, por algo me desarrollé pero por el otro lado también quiero satisfacer esa necesidad biológica de tener un bebé" (Ing. Civil Industrial en Electrónica, 27 años). 
"El único problema que yo veo es que como más a futuro dejar de lado la familia, siempre se ve a las mamás que cumplen una función maternal más importante y que el papá es el que siempre sale a trabajar..." (Ingeniería Civil de Minas, 18 años).

Por último, en la quinta categoría, "Carreras con Alta Dificultad", se evidenciaron diferencias entre las estudiantes universitarias y técnicas que cursan primero y aquellas que están en el último año de la carrera. El primer grupo muestra en sus discursos lo que Eccles y otros (1983) denomina Modelo de Elección de Logro, jugando un rol relevante en la elección de carrera, pues se conjugan las creencias, los estereotipos, ideologías, las experiencias personales, entre otras, y aunque ejercen un efecto indirecto, son significativas en las elecciones que los sujetos realizan. En este sentido, las estudiantes de primer año destacan en sus discursos las dificultades que ellas percibían al ingresar a la carrera: estudiar mucho, adaptarse a los turnos, usar casco y llenarse de tierra, usar zapatos de seguridad, la idea de que no era carreras femeninas, no confiaban en sus capacidades", pensaban que iban a andar llenas de grasa (motor), que tenían que buscar minerales o que iba a ser una carrera más práctica. En cambio en el grupo de estudiantes de último año, estas dificultades se disipan, ya que ellas visualizan la infraestructura de las carreras como insuficientes. Por ejemplo, laboratorios en la universidad y, en el caso de las estudiantes técnicas, son asignaturas de matemáticas la dificultad central. Algunos discursos que ejemplifican lo expuesto.

"Mira al principio un poco difícil, más encima las matemáticas como que no me gustaban mucho, estuve un año haciendo nada, entonces, como que perdí el ritmo de estudiar, el primer semestre fue un poco difícil" (Geominería, 25 años).

"Me costó harto volver a abrir los cuaderno buscar las matemáticas, uhh terrible (risas)" (Maquinaria Pesada, 25 años). A continuación la Tabla №2 sintetiza los hallazgos presentados. La tabla 3 resume lo expuesto

Tabla 3: Matriz de análisis estudiantes de primer y último año de carreras mineras técnicas y universitarias

\begin{tabular}{|c|c|c|c|c|}
\hline \multirow{3}{*}{$\begin{array}{l}\text { Categoría } \\
\text { abierta- } \\
\text { conceptual }\end{array}$} & \multicolumn{4}{|c|}{ Categoría central : visión sobre carreras del área minera } \\
\hline & \multicolumn{2}{|c|}{ Universitarias } & \multicolumn{2}{|c|}{ Técnicas } \\
\hline & $\begin{array}{l}\text { primer año } \\
\text { subcategoría }\end{array}$ & $\begin{array}{c}\text { último año } \\
\text { subcategoría }\end{array}$ & $\begin{array}{l}\text { primer año } \\
\text { subcategoría }\end{array}$ & $\begin{array}{l}\text { último año } \\
\text { subcategoría }\end{array}$ \\
\hline 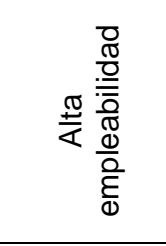 & $\begin{array}{l}\text { Hay trabajo } \\
\text { Muchas proyecciones } \\
\text { laborales } \\
\text { Altos ingresos }\end{array}$ & $\begin{array}{l}\text { Rentabilidad de la carrera } \\
\text { Hay trabajo }\end{array}$ & $\begin{array}{l}\text { Oportunidades } \\
\text { Laborales } \\
\text { Opciones de empleo } \\
\text { Altos ingresos }\end{array}$ & $\begin{array}{l}\text { Rentabilidad de la } \\
\text { carrera } \\
\text { La minería es muy } \\
\text { rentable } \\
\text { Aspirar a tener mejor } \\
\text { futuro }\end{array}$ \\
\hline 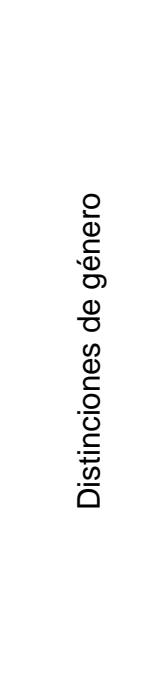 & $\begin{array}{l}\text { Los hombres tienen } \\
\text { más capacidad para } \\
\text { las matemáticas } \\
\text { Ellos se manejan } \\
\text { mejor } \\
\text { Los hombres tienen } \\
\text { más experiencia }\end{array}$ & $\begin{array}{l}\text { Igualdad de capacidades } \\
\text { No hay diferencia de } \\
\text { género } \\
\text { Reafirmación de } \\
\text { características orientadas } \\
\text { a los roles } \\
\text { Incremento de mujeres } \\
\text { en sus carreras } \\
\text { Las mujeres son más } \\
\text { ordenadas, responsables, } \\
\text { el hombre más } \\
\text { desordenado } \\
\text { Hay que pararse de igual } \\
\text { a igual con los hombres } \\
\text { Falta de incentivos para } \\
\text { la incorporación de la } \\
\text { mujer a estas carreras }\end{array}$ & $\begin{array}{l}\text { Los hombres tienen más } \\
\text { conocimiento } \\
\text { Los compañeros vienen de } \\
\text { un liceo técnico } \\
\text { Tienen más experiencia en } \\
\text { el área }\end{array}$ & $\begin{array}{l}\text { Igualdad de } \\
\text { capacidades } \\
\text { Tienen las mismas } \\
\text { capacidades y } \\
\text { pueden hacer las } \\
\text { mismas cosas }\end{array}$ \\
\hline
\end{tabular}


Tabla 3 (continuación)

\begin{tabular}{|c|c|c|c|c|}
\hline 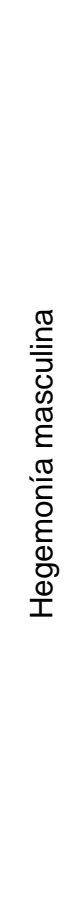 & $\begin{array}{l}\text { Competir con } \\
\text { hombres. } \\
\text { Mujer en minería } \\
\text { aún es tabú. } \\
\text { Se prefiere a } \\
\text { hombres por su } \\
\text { fuerza física. } \\
\text { Mujeres con falta de } \\
\text { disponibilidad por } \\
\text { embarazo. } \\
\text { Ahorro de costos al } \\
\text { contratar a } \\
\text { hombres. } \\
\text { Hombres con mayor } \\
\text { disponibilidad. } \\
\text { País machista } \\
\text { Hay asedio } \\
\text { masculino } \\
\text { Prejuicios sobre } \\
\text { personas con } \\
\text { menor nivel de } \\
\text { estudios }\end{array}$ & $\begin{array}{l}\text { Reafirmación de carreras } \\
\text { masculinas y adaptación } \\
\text { al entorno } \\
\text { Mujeres deben enfrentar } \\
\text { un ambiente masculino } \\
\text { Carrera muy masculina } \\
\text { de alto riesgo } \\
\text { Se necesita fuerza } \\
\text { Cómo te miran } \\
\text { Hablan con garabato, son } \\
\text { groseros } \\
\text { Hay que hacerse respetar }\end{array}$ & $\begin{array}{l}\text { Competir con el hombre } \\
\text { Discriminación por fuerza } \\
\text { física } \\
\text { Recibir menor } \\
\text { remuneración } \\
\text { Sistema biológico, } \\
\text { hormonas, menstruación } \\
\text { Intimidación por trabajar } \\
\text { con hombres desconocidos } \\
\text { Desconfianza de sus } \\
\text { intenciones }\end{array}$ & $\begin{array}{l}\text { Reafirmación de } \\
\text { carreras masculinas y } \\
\text { adaptación al entorno } \\
\text { Acostumbrarse a los } \\
\text { hábitos masculinos es } \\
\text { difícil } \\
\text { La gente siempre se } \\
\text { sorprende que } \\
\text { estudie maquinaria } \\
\text { Desconfianza porque } \\
\text { es carrera de } \\
\text { hombres }\end{array}$ \\
\hline 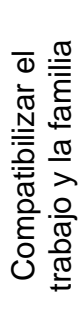 & $\begin{array}{l}\text { Dejar la familia por } \\
\text { el trabajo } \\
\text { Ser mamá y estar } \\
\text { en la minería } \\
\text { Es el mayor } \\
\text { problema } \\
\text { desprenderse de } \\
\text { la familia }\end{array}$ & $\begin{array}{l}\text { Limitan la } \\
\text { compatibilización de roles } \\
\text { Madre trabajadora } \\
\text { No les gusta contratar } \\
\text { mujeres por posibles } \\
\text { embarazos }\end{array}$ & $\begin{array}{l}\text { La maternidad es lo más } \\
\text { difícil } \\
\text { Es costoso dejar todo por } \\
\text { la familia } \\
\text { Es mejor no tener hijos } \\
\text { para mantener la } \\
\text { independencia }\end{array}$ & $\begin{array}{l}\text { La maternidad es un } \\
\text { problema }\end{array}$ \\
\hline 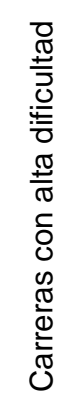 & $\begin{array}{l}\text { Estudiar mucho } \\
\text { Adaptación a } \\
\text { turnos } \\
\text { Usar bototos } \\
\text { Usar casco y estar } \\
\text { llena de tierra } \\
\text { Nada femenino } \\
\text { No confiaba en mis } \\
\text { capacidades }\end{array}$ & $\begin{array}{l}\text { Falta implementación } \\
\text { (laboratorio) }\end{array}$ & $\begin{array}{l}\text { Pensé que iba andar llena } \\
\text { de grasa } \\
\text { Pensé que tenía que } \\
\text { buscar minerales, no más } \\
\text { Pensé que iba a ser una } \\
\text { carrera más práctica }\end{array}$ & $\begin{array}{l}\text { Dificultad con las } \\
\text { matemáticas }\end{array}$ \\
\hline
\end{tabular}

\section{Expectativas sobre el futuro laboral}

En esta tercera categoría central analizada las estudiantes que cursan el último año de carreras, se reiteran en los discursos la fuerza de los estereotipos de género en los dos sub temas identificados: la Hegemonía Masculina y Compatibilizar el Trabajo y Familia. Ambos se configuran como tensiones frente a las expectativas laborales para incorporarse al área minera.

La Hegemonía Masculina fue abordada por las estudiantes universitarias y técnicas en dos dimensiones, la primera referida a las características de los espacios laborales predominantemente masculinos, donde ellas deben estar preparadas e inclusive a la defensiva para contrarrestar el dominio de los hombres. Las estudiantes universitarias precisan: "hay que estar preparadas para enfrentar a los hombres", "son pesados, hablan fuerte", "siempre quieren pisotear", "hacemos el mismo trabajo y ganamos menos". Las estudiantes técnicas son inclusive más explicitas en expresar: "hay que tener carácter fuerte para trabajar en esto", "hay que ubicar al machista (hombre) que te quiere pasar a llevar" y "la menor capacidad de las mujeres para hacer trabajo de fuerza física". La segunda dimensión, relacionada con los estereotipos de género, se expresa: "las mujeres son más organizadas", "planificadas", "ordenadas" y "detallistas". 
"[...] me siento preparada para enfrentar a los hombres, pero creo que todo va en el carácter o actitud de la mujer, porque igual hay mujeres que son muy delicadas, como que uno les dice algo y lloran y otras que están preparadas para eso, porque los hombres son pesados, hablan fuerte y siempre te van a querer pisotear porque van a querer ser mejor que tú" (Ing. Civil Industrial en Minas, 27 años).

“Las mujeres tienen que... para trabajar en esto yo creo que igual uno tiene que tener su carácter para saber ubicar al que no... al machista que te quiera bajar de perfil o tratarte de pasar a llevar, porque voy a estar con puros hombres" (Maquinaria Pesada, 25 años). La tabla 3 sintetiza los hallazgos.

En la segunda categoría presente en los discursos analizados, Compatibilizar el Trabajo y Familia, se muestra dos aspectos a considerar. El primero y más presente corresponde a las estudiantes universitarias de último año; para ellas la maternidad es lo más difícil de compatibilizar con el trabajo, ya que es considerado un obstáculo en sus expectativas laborales. Señalan que es injusto tratar de compatibilizar el desarrollo profesional con la maternidad y que sus expectativas laborales sean limitadas por lo mismo. En el caso de las jóvenes de carreras técnicas, manifiestan su interés en seguir estudiando y luego pensar en tener hijos, y evalúan la fertilidad femenina como un problema para los empleadores.

"Uno tiene expectativas laborales más limitadas por el hecho de que vamos a tener que ser mamás (...) Si es que tienen a dos profesionales, hombre y mujer de la misma universidad y con las mismas notas, no va a importar como sea su entrevista personal si no que va a importar más si es que es hombre o mujer, porque contratar a un hombre es mucho más sencillo" (Geología, 23 años).

"Todo te cuesta mucho más, porque tienes que sacrificar mucho los tiempos de tu hijo (...) me he perdido muchas cosas de él y porque llego y siempre estoy cansada (...) cuesta porque te pueden echar si quedas embarazada, cuesta porque tienes que tener un día con 30 horas no con 24, porque no me alcanza el tiempo y el sacrificio físico-mental que significa tener un hijo y hacerte responsable de él (Mecánica Automotriz en Sistemas Electrónicos, 23 años).

Y en una segunda dirección destacan, al igual que en la categoría anterior, los estereotipos de género donde las estudiantes reconocen su habilidades y las valoran, aunque estas características sean la manifestación de la socialización familia y escolar, donde se refuerza la segregación de género.

"Nosotras somos mucho más organizadas porque desde chicas somos así (...) somos planificadoras, mucho más ordenadas, y esos aspectos a la hora de trabajar, la gente, las personas que te contratan lo toman mucho en cuenta." (Mecánica Automotriz en Sistemas electrónicos, 30 años).

"Lo que se está viendo es que yo voy a hacer la misma pega que un hombre, pero yo voy a recibir menos sueldo que él, por la misma pega por ejemplo a mí me ofrecen como el $20 \%$ menos que él, por el puro hecho de ser mujer" ( Ing. Civil Mina, 22 años).

En ambas categorías, Hegemonía Masculina y Compatibilizar el Trabajo y Familia, la condición de mujer es distinguida en los discursos como diferencias importantes respecto a los hombres, pues son ellas las que tienen que lidiar con el predominio masculino presente en la minería y también las que perciben los obstáculos que implica compatibilizar el trabajo con la familia. La tabla 4 resume lo expuesto.

Tabla 4: Matriz de análisis entrevistas a estudiantes de último año en carreras mineras universitarias y técnicas

\begin{tabular}{|c|c|c|}
\hline \multirow{2}{*}{$\begin{array}{l}\text { Categoría } \\
\text { Abierta } \\
\text { Conceptual }\end{array}$} & \multicolumn{2}{|c|}{ Categoría central: expectativas sobre futura inserción laboral } \\
\hline & $\begin{array}{l}\text { universitarias } \\
\text { último año } \\
\text { sub categoríal }\end{array}$ & $\begin{array}{c}\text { técnicas } \\
\text { último año } \\
\text { sub categoría }\end{array}$ \\
\hline 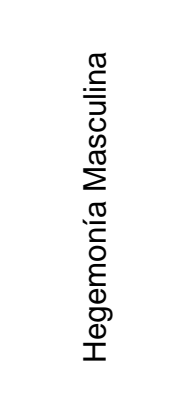 & $\begin{array}{l}\text { Hay que estar preparada para enfrentar } \\
\text { a los hombres } \\
\text { Ellos son pesados hablan fuerte, } \\
\text { siempre te quieren pisotear } \\
\text { Hacer el mismo trabajo, pero recibir } \\
\text { menos remuneración cerca de un } 20 \% \\
\text { menos } \\
\text { Las mujeres son más ordenadas }\end{array}$ & $\begin{array}{l}\text { Hay que tener carácter fuerte para trabajar en esto } \\
\text { Hay que ubicar al machista que te quiere pasar a } \\
\text { llevar } \\
\text { La menor capacidad de la mujer para hacer trabajo } \\
\text { de fuerza } \\
\text { Hay que demostrar que puedes hacer el trabajo igual } \\
\text { que un hombre } \\
\text { Las mujeres son más organizadas, planificadas y } \\
\text { ordenadas } \\
\text { Son más minuciosas y detallistas } \\
\text { Las mujeres son más delicadas }\end{array}$ \\
\hline
\end{tabular}


Tabla 4 (continuación)

\begin{tabular}{|c|c|c|}
\hline 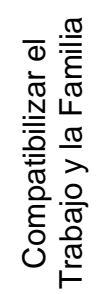 & $\begin{array}{l}\text { Es injusto tratar de compatibilizar el } \\
\text { desarrollo profesional y la maternidad } \\
\text { Expectativas laborales más limitadas por } \\
\text { la maternidad } \\
\text { La maternidad es un obstáculo contratar } \\
\text { a hombres es más sencillo } \\
\text { Prioridad desarrollar la carrera } \\
\text { La empresa lo ve como una perdida }\end{array}$ & $\begin{array}{l}\text { Quiero seguir estudiando, después pensar en tener } \\
\text { hijos } \\
\text { La fertilidad femenina es un problema para los } \\
\text { empleadores } \\
\text { Es difícil compatibilizar la familia y el trabajo es } \\
\text { mucho el desgaste físico-mental } \\
\text { Me gusta el sistema de turno, se puede viajar } \\
\text { Prefieren a los hombres }\end{array}$ \\
\hline
\end{tabular}

\section{DISCUSIÓN FINAL}

Este estudio evidencia que las motivaciones, visiones $\mathrm{y} / \mathrm{o}$ expectativas fueron aspectos que se interrelacionaron a lo largo de los discursos de las jóvenes, conformando el corpus central de la presente investigación. En los resultados se distingue por una parte que existen un conjunto de transformaciones y tensiones, respecto a la proyección de las estudiantes en el rubro minero (Ver Fig. 1)

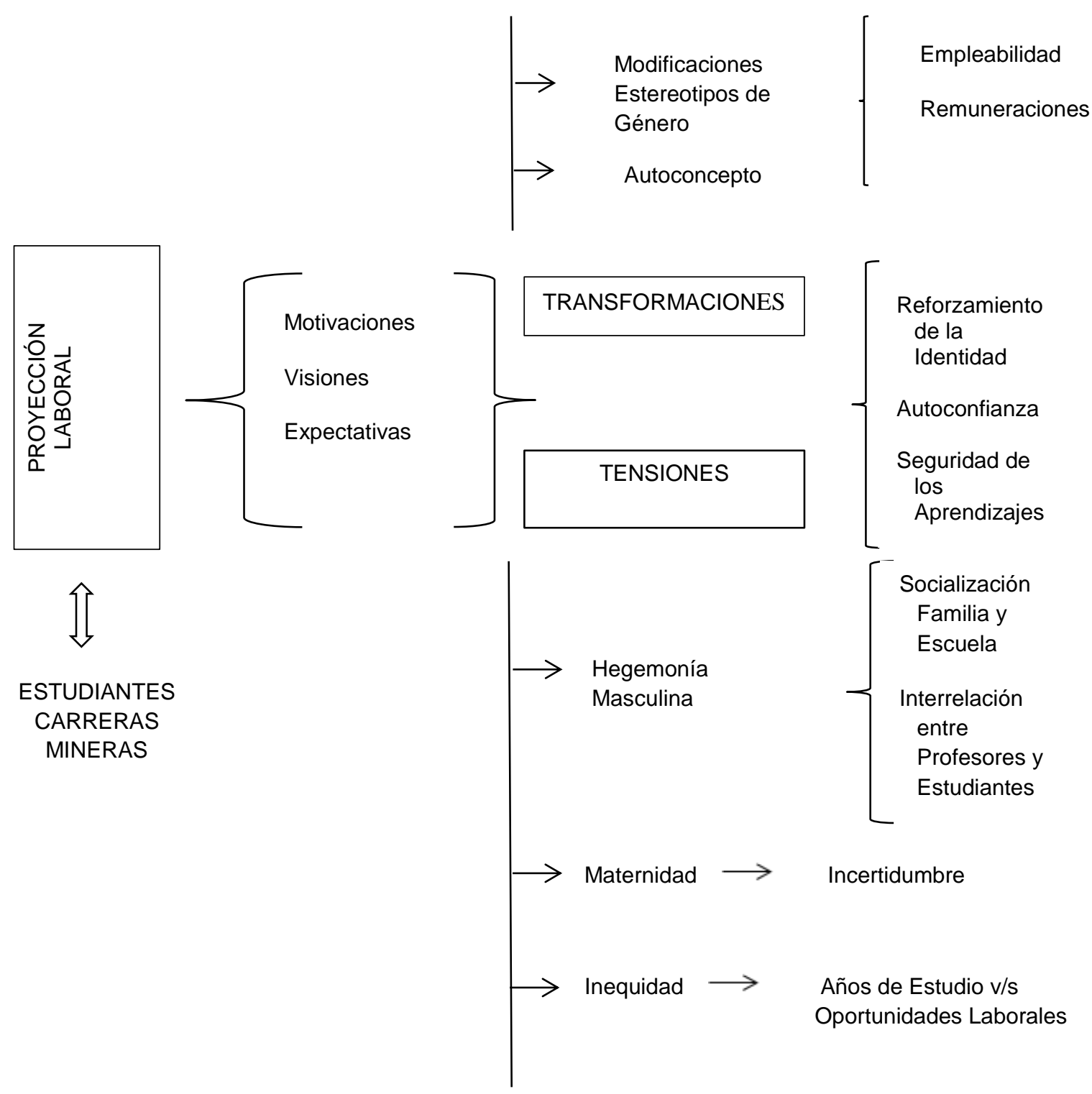

Fig. 1: Síntesis principales hallazgos 
Este estudio evidencia que las motivaciones, visiones $\mathrm{y} / \mathrm{o}$ expectativas fueron aspectos que se interrelacionaron a lo largo de los discursos de las jóvenes, conformando el corpus central de la presente investigación. En los resultados se distingue por una parte que existen un conjunto de transformaciones y tensiones, respecto a la proyección de las estudiantes en el rubro minero (Fig. 1)

Entre las transformaciones destacan modificaciones en los estereotipos de género. En la actualidad, las estudiantes valoran la mayor empleabilidad y las altas remuneraciones que ofrece el rubro, motivaciones que eran inexistentes en generaciones anteriores, producto de las restricciones legales que limitaba el acceso de las mujeres a los yacimientos. Junto al aspecto económico, el auto concepto que tienen las jóvenes de sí mismas, reflejado en una mayor confianza en sus capacidades y en las habilidades personales que poseen son importantes. De este modo, se confirma la centralidad de las identidades personales en ambos grupos entrevistados, donde el autoconcepto desarrollado en el modelo de Eccles, Barber y Jozefowicz, (1999) juega un rol central, ya que les permite sobrepasar las dificultades que enfrentan en la formación profesional, sea en las universidades o instituciones técnicas. Asimismo, como lo expone estos autores, el conjunto de creencias, símbolos e ideologías, influyen en la construcción y estereotipos de género existentes en las mujeres, en los hombres y en la comunidad en general.

También se destacan los cambios que vivencian las estudiantes durante la formación profesional, lo que se expresa en que aquellas que cursan los últimos años de carrera acentúan su autoconfianza y se sienten seguras de los aprendizajes que han alcanzado, proyectándose con mayor certeza en el mercado laboral.

Por otro lado, también los hallazgos muestran las tensiones presentes en este proceso. Desde el inicio hasta el término de sus carreras, las jóvenes perciben la fuerza de la hegemonía masculina presente en el mercado minero, que se incuba en las aulas de las universidades e institutos desde el principio de la formación profesional. La hegemonía masculina se expresa de distinta manera: en el menor número de mujeres en los diferentes cursos; en las interrelaciones entre estudiantes y profesores con algunos comentarios que reciben de los docentes, quienes dudan de las capacidades de las jóvenes para trabajar en el sector; en las relaciones con los compañeros durante la carrera; en las dificultades para obtener cupos para práctica profesionales en forma temprana en las diferentes empresas del rubro, entre otras. Este conjunto de tensiones se traspasa generacionalmente y se valida como constitutiva de la cultura organizacional del sector; más aún, se reproduce en las distintas etapas de formación del capital humano especializado para el rubro.

Otro hallazgo se relaciona con la centralidad que las estudiantes asignan en los discursos a la maternidad. Al respecto, ellas manifiestan una alta incertidumbre sobre las posibilidades reales que tendrán para compatibilizar el trabajo en el rubro minero con la familia, inclusive las jóvenes prefieren renunciar, al menos por un tiempo, a los hijos. Estos argumentos son ambivalentes en cuanto al grado real de libertad que conlleva esta decisión o es sólo una estrategia de ellas para evitar la tensión que la maternidad les genera, ya que no se visualizan cambios relevantes en las empresas en favor de compatibilizar la labor de las trabajadoras, menos de los trabajadores con la familia, ni tampoco una participación más activa de los hombres en las tareas de cuidado de los hijos, en lo que al sector minero se refiere (Salinas y Romaní, 2014).

Estos resultados son coincidentes con el estudio realizado por Sarrió, Barbera, Ramos y Candela (2002) en el que comprobaron que los estereotipos de género, las responsabilidades familiares asumidas mayoritariamente por la mujer y la carencia de soportes y ayudas sociales en el cuidado de los hijos, provoca que ellas sean excluidas de aquellas actividades más desafiantes y claves en las organizaciones. Respecto al género y la discriminación, López-Sáez, Lisbona y Sáinz (2004), plantean que desde que nacen, se asigna a las niñas características distintas que a los niños, a pesar que no cumplan ningún rol, ya que el carácter prescriptivo o deseable que se atribuye al estereotipo implica que se establecen cómo deben ser y comportarse las personas. Y con ello, se cumple la función de dejar las cosas tal como están, al categorizar y justificar el prejuicio y la discriminación.

Las entrevistadas perciben una situación de inequidad respecto a los años que estudian y a los esfuerzos que ello implica, sobre todo porque no existe una motivación temprana en la escuela y/o familia por proyectarlas en esta área; además de las dificultades que luego experimentan para insertarse a este mercado laboral como profesionales. Por otra parte, los resultados permiten visualizar la fuerza de la socialización familiar y escolar y como se relaciona con la posterior proyección laboral que hacen las estudiantes. A pesar de los cambios que han posibilitado el ingreso de las mujeres al mercado laboral a nivel internacional y nacional, el acceso a los medios de comunicación y con ello a patrones y modelos culturales globalizados, al interior de la familia y de la escuela se incuban y reproducen estereotipos de género tradicionales, que no se ajustan al escenario de transformaciones que han experimentado las sociedades occidentales, retrotrayendo a las mujeres al mundo privado y a la idealización de la familia nuclear patriarcal, donde ellas juegan un rol central, limitándose la democratización de las relaciones al interior de la familia. 


\section{CONCLUSIONES}

De lo analizado e investigado en este trabajo se pueden extraer las siguientes conclusiones principales, relacionadas con la proyección laboral y las condiciones de empleabilidad que perciben las estudiantes de carreras mineras

La proyección laboral de las estudiantes de carreras mineras esta mediada por un conjunto de tensiones que experimentan desde el inicio hasta el término de sus carreras, asociadas a la hegemonía masculina del sector, y que marca tempranamente la cultura organizacional de las universidades y centros de formación técnica, ya sea a través de los discursos entre los/as estudiantes, las relaciones con los profesores, las experiencias prácticas tempranas, la escasa dotación de mujeres docentes, como por el conjunto de prejuicios y estereotipos sexistas que circulan en el sistema educacional, comprobándose de este modo la hipótesis que se planteó en el presente estudio, es decir que existen diferencias entre la proyección laboral de las estudiantes y las condiciones de empleabilidad que enfrentan en la industria

Las estudiantes de carreras mineras durante la formación profesional, contrastan críticamente los modelos de socialización familiar y escolar que han recibido, conformándose en barreras de carrera, ya que obstaculizan el desafío que se implica incorporarse a un área, aún, de predominio masculino.

La hegemonía masculina predominante en la cultura organizacional minera, refuerza la ambivalencia en las estudiantes, frente a la incertidumbre que les provoca compatibilizar la familia y el trabajo, con las condiciones de empleabilidad del sector, evidenciándose los escasos o poco significativos que han sido en términos generales, las transformaciones que se han implementado en las diferentes empresas mineras, en favor de alcanzar una minería más moderna e inclusiva.

Por último, la implicancia del estudio es introducir a la educación superior perspectiva de género, y con ello mejorar las competencias genéricas que se esperan de los profesionales en pleno siglo XXI, potenciando la proyección laboral de las mujeres, especialmente en mercados laborales masculinos, cuestión pendiente tanto a nivel nacional como latinoamericano

Finalmente, algunas de las limitaciones de este esta investigación se relacionan con la necesidad de ampliar las percepciones a los estudiantes varones. Estudios futuros, en esta misma línea, podrían incorporar a los hombres para conocer sus percepciones. Igualmente, podrían entrevistarse a mujeres y hombres trabajadores en la industria y así enriquecer esta línea investigativa.

\section{AGRADECIMIENTOS}

Los autores agradecen al proyecto Fondecyt $N^{\circ} 1150055$, por haber financiado el estudio que dio origen al presente trabajo.

\section{REFERENCIAS}

Alonso, L., La mirada cualitativa en sociología 2da Edición, Editorial Fundamentos, España (2003)

Araujo, K. y Martuccelli, D., Desafíos comunes. Retrato de la sociedad chilena y sus individuos. Tomo II, Trabajo, sociabilidades y familias. Santiago de Chile, Serie Individuo y Ciencias Sociales LOM Ediciones (2012)

Área Minera, 2015, https://goo.gl/obNzyW. Acceso: 15 de junio (2015)

Arriagada, I., Changes and Inequalities in Latin American Families. In The Wiley Blackwell companion to the sociology of families. Edited by Judith Treas, Jacqueline Scott, and Martin Richards, Wiley-Blackwell, 83-106 (2014)

Ayala, E., Mujer y minería: situación de la industria chilena (2012)

Benítez, L., "La perspectiva de género en comunicación y desarrollo". En Comunicación y Desarrollo. Prácticas comunicativas y empoderamiento local. Serie Comunicación Latina, España: Editorial Gedisa, 201-239 (2012)

Bester, J., The perception of career barriers among South African university students. Thesis presented in fulfilment of the requirements for the degree of Master of Arts (Psychology) in the Faculty of Arts and Social Sciences at Stellenbosch University (2011)

Cardoso, P. y Marques F., Perception of career barriers: The importance of gender and ethnic variables, 
International Journal of Educational / Vocational Guidance, 8, 49-61 (2008)

Consejo Minero, Fuerza Laboral de la Gran Minería Chilena 2012-2020. Diagnóstico y recomendaciones, 2012, https://goo.gl/WOaBiM. Acceso: 10 de mayo (2015)

Consejo Minero, Reporte Anual Consejo Minero 2013-2014 (2014)

Denzin, N., y Lincoln, Y., El campo de la investigación cualitativa. Manual de investigación cualitativa, Vol.1, Editorial Gedisa, España (2012)

Donoso, T., Figuera, P., y Rodríguez Moreno, M., Barreras de género en el desarrollo profesional de la mujer universitaria, doi: 10-4438/1988-592X-RE-2011-355-021, Revista Educación, 355, 187-212 (2011)

Eccles, J., Adler, T., Futterman, R., Goff, S., Kaczala, C., Meece, J. y Midgley, C., Expectations, values and academic behaviors. En J.T. Spence. Perspective on achievement and achievement motives. Psychological and sociological approaches. San Francisco, EEUU, Freeman, 75-146 (1983)

Eccles, J., Barber, B. y Jozefowicz, D., Linking gender to educational, occupational, and recreational choices: Applying the Eccles et al. Model of achievement-ralted choices. En W. B. Swann, J.H. Langlois \& L.A. Gilbert. Sexism and stereotype in modern society. Washintong D.C., EEUU: American Psychological association, 153-191 (1999)

Encuesta de Caracterización Socioeconómica Nacional, CASEN 2013, https://goo.gl/V4qoWG. Acceso: 5 de abril (2016)

Espejo, R., Interacción Simbólica en un Acto de Subjetividad de Género, doi: 10.4067/S071850062015000500006, Formación Universitaria 8(5) 43-58 (2015)

Espino, A., Perspectivas teóricas sobre género, trabajo y situación del mercado laboral latinoamericano. La economía feminista desde América Latina. Una hoja de ruta sobre los debates actuales en la región. ONU Mujeres, Santo Domingo, República Dominicana, 2012, https://goo.gl/xYGNcG. Acceso: 15 de mayo (2015)

Harding, S., The Science Questions in Feminism. Ithaca and London Cornell University Press (1986)

Ibañez, M., Al otro lado de la segregación ocupacional por sexo. Hombres en ocupaciones femeninas y mujeres en ocupaciones masculinas, doi:10.3989/ris.2008.04.14, Revista Internacional de Sociología, 68 (1), 145-164 (2010)

Instituto Nacional de Estadística, Situación laboral de las mujeres ocupadas y las brechas salariales que inciden en la estructura ocupacional chilena Análisis de Género y Mercado del Trabajo (2013)

International Women and Mining Network (IWIM), Women affected by mining, de WIM Community Portal Ltd and Barbara Dischinger, 2014, https://goo.gl/erjuCz. Acceso: 30 Octubre (2014)

Levy-Leboyer, C., La Gestión de las Competencias, Barcelona, Ediciones Gestión (1997)

Lisbona, A. y Topa, G., Trabajando en la diversidad: inserción profesional de la mujer. En F. Palací y J. Moriano, El nuevo mercado laboral. Estrategias de inserción y desarrollo de inversión. Madrid, España, Universidad Nacional de Educación a Distancia, 229-259 (2003)

López-Sáez, M., Modelos de elección de estudios en la revisión de la literatura. En M. López-Sáez, La elección de una carrera típicamente femenina o típicamente masculina, 1a ed., Madrid, España, Centro de investigación y documentación, 21-38 (1995)

López-Sáez, M., Expectativa de rol Profesional de mujeres estudiantes de carreras típicamente femeninas y típicamente masculinas, ISSN 1578-908X, Acción Psicológica, 3,2, 111-123 (2004)

López-Sáez, M., Lisbona, A. y Sáinz, M., Mujeres ingenieras: percepciones sobre su vida profesional, Revista de Psicología General y Aplicada, 57, 161-180 (2004)

Mi Futuro, \#TuFuturo en Minería y Extracción 2014, https://goo.gl/nQqxt1. Acceso: 26 de diciembre (2016)

Ministerio de Educación, Mineduc, https://goo.gl/f7dQKX. Acceso: 26 de diciembre (2016)

Morales, J.F., y Cuadrado, I., Algunas claves sobre el techo de cristal en las organizaciones, Revista de Psicología del Trabajo y de las Organizaciones, 23(2), 183-202 (2007)

Moriano, J., Palací, F., y Morales, J. Adaptación y validación en España de la escala de Autoeficacia 
Emprendedora, Revista de Psicología Social, 21(1), 51-64 (2006)

Mosterio, M., El género como factor condicionante de la elección de carrera: hacia una orientación para la igualdad de oportunidades entre sexos. Revista Galeno-Portuguesa de psicoloxía e educación, ISSN 11381663, https://goo.gl/WXcB2c. Acceso: 12 de Mayo (2015), 1, 305-315 (1997)

Navarro-Astor, E., Román-Onsalo, M., e Infante-Perea, M., Revisión internacional de estudios de barreras de carrera bajo la perspectiva de género en la industria de la construcción, Innovar 26(61), 103-118 (2016)

Organización Internacional del Trabajo (OIT). LABORSTA. Base de datos sobre estadísticas del trabajo, 2014, https://goo.gl/zizOlw. Acceso: 25 de abril (2015)

Olavarría, J., y Céspedes, C. (Eds.). Trabajo y familia: ¿conciliación? Perspectivas de género. Santiago, CEM, SERNAM, FLACSO (2002)

Organización para la Cooperación y el Desarrollo Económicos, OCDE, 2015, https://goo.gl/G4i8n1. Acceso: 3 de septiembre (2016)

Painter-Morland, M., Género Liderazgo y Organización, 2012, https://goo.gl/OaCAKm. Acceso: 28 de octubre (2014)

Ramos, A., Sarrió, M., Barberá, E. y Candela, C., Mujeres directivas y demandas organizacionales, Revista de Psicología Social, 17(2), 183-192 (2002)

Ruiz, J., Metodología de investigación cualitativa, Universidad de Deusto, Bilbao, España (1996)

Salinas, P., y Cárdenas, J., Métodos de Investigación Social, Intiyan ediciones Ciespal, Quito, Ecuador (2009)

Salinas, P., Discourses as Control Devices in the Mining Culture: Tensions in the Integration of Women in Chilean Mining International, doi 1932-8036/2013FEA0002, Journal of Communication 7, 1835-1851 (2013)

Salinas, P., y Romaní, G., Gender barriers in Chilean mining: a strategic management, doi: 10.1108/ARLA11-2013-0184, Academia Revista Latinoamericana de Administración, 27(1), 92-107 (2014)

Salinas, P., y Romaní, G., Hegemonía masculina, freno en equipos mixtos en la minería chilena, Revista Mexicana de Sociología, 78(3) 469-496 (2016)

Sáinz, M., López-Sáez, M., y Lisbona, A., Expectativas de rol profesional de mujeres estudiantes de carreras típicamente femeninas o masculinas, Acción Psicológica, 3 (2), 111-123 (2004)

Sarrió, M., Barberá, E., Ramos, A., y Candela, C., El techo de cristal en la promoción profesional de las mujeres, Revista de Psicología Social: International Journal of Social Psychology, 17 (2), 167-182 (2002)

Scott, J., Gender: A Useful Category of Historical Analysis, https://goo.gl/MiEqhF. Acceso: 10 de Noviembre (2012). The American Historical Review, 91 (5), 1053-1075 (1986)

Servicio de Información de Educación Superior (SIES), Principales Indicadores de Educación Superior en Perspectiva de Género, 2014, https://goo.gl/m86W4G. Acceso: el 22 de mayo (2014)

Strauss, A. y Corbin, J., Bases de la Investigación Cualitativa. Técnicas y procedimientos para desarrollar la Teoría Fundamentada. Editorial Universidad de Antioquia, Colección Contus, Medellín, Colombia (2002)

Swanson, J., Daniels, K y Tokar, D., Assessing perceptions of career related barriers: The Career Barriers Inventory. Journal of Career Assessment, 4(2), 219-244 (1996)

Swanson, J. y Woitke, R., Theory Into Practice in Career Assessment for Women: Assessment and Interventions Regarding Perceived Career Barriers, Journal of Career Assessment September 5,443-462 (1997)

Trinidad, A., Carrero, V., y Soriano, R., Teoría fundamentada Grounded Theory. La construcción de la teoría a través del análisis interpretacional. Centro de Investigación Sociológica (CIS). Cuadernos Metodológicos 37, España (2006)

Unesco, Declaración Mundial Sobre la Educación Superior en el Siglo XXI: Visión y Acción Conferencia Mundial Sobre la Educación Superior. Visión y acción, 1998, https://goo.gl/LYqQK1. Acceso: 17 de abril (2016)

Uribe- Echeverría, V., Inequidad de Género en el Mercado Laboral: El rol de la división sexual del trabajo. Cuadernos de Investigación № 35. Santiago, Chile: División de Estudios de la Dirección del Trabajo, 2008, https://goo.gl/hUd1sa. Acceso: 15 de octubre (2012) 